\title{
The Effect of Low Temperature Cryocoolers on the Development of Low Temperature Superconducting Magnets*
}

\author{
M. A. Green \\ Lawrence Berkeley National Laboratory, Berkeley CA 94720, USA
}

\begin{abstract}
The commercial development of reliable $4 \mathrm{~K}$ cryocoolers improves the future prospects for magnets made from low temperature superconductors (LTS). The hope of the developers of high temperature superconductors (HTS) has been to replace liquid helium cooled LTS magnets with HTS magnets that operate at or near liquid nitrogen temperature. There has been limited success in this endeavor, but continued problems with HTS conductors have greatly slowed progress toward this goal. The development of cryocoolers that reliably operate below $4 \mathrm{~K}$ will allow magnets made from LTS conductor to remain very competitive for many years to come. A key enabling technology for the use of low temperature cryocoolers on LTS magnets has been the development of HTS leads. This report describes the characteristics of LTS magnets that can be successfully melded to low-temperature cryocoolers. This report will also show when it is not appropriate to consider the use of low-temperature cryocoolers to cool magnets made with LTS conductor. A couple of specific examples of LTS magnets where cryocoolers can be used are given.
\end{abstract}

\section{INTRODUCTION}

A major impediment to the development of commercial superconducting magnet has been the cooling of those magnets. For many applications, superconducting magnets are cost competitive with conventional magnet technology. Yet the superconducting solution has been rejected because of the perceived problems with cooling. Industrial users of superconducting magnets are not interested in transferring either liquid helium or liquid nitrogen, and they consider the need to do so to be undesirable.

HTS conductors were supposed to allow superconducting magnets to move into the market place within a few years of their discovery. This has not been the case for a couple of reasons, which are as follows: 1) HTS conductors are not truly superconductors in the way that niobium titanium is until the temperature is well below the critical temperature of the conductor. 2) HTS materials have rather low critical current densities at liquid nitrogen temperature and the critical current density drops rapidly when a magnetic field is applied to the conductor. In order for the critical current to be increased at higher fields, the temperature of the conductor has

Manuscript received 18 September 2000.

* This Research is supported by the Office of Science US Department of Energy under contract number DE-AC0376SF00098 to be reduced to temperature substantially below that of liquid or even solid nitrogen. 3) HTS materials do exhibit strong field anisotropy. In real magnets, there are always field lines that penetrate the conductor in the bad direction. 4) HTS materials are brittle, so mechanical support is a real issue. 5) HTS materials are expensive. The cost of HTS material per ampere meter is orders of magnitude higher than for $\mathrm{Nb}-\mathrm{Ti}$ at $4 \mathrm{~K}$ when used at a magnetic induction below $7 \mathrm{~T}$.

Niobium titanium magnets have traditionally required that liquid helium in some form be used as a coolant. This has been a major impediment to the development of commercial superconducting magnets, except in the field of MRI. Until recently, most MRI magnets have been cooled using stored liquid helium and liquid nitrogen. Large niobium titanium magnets have been cooled using Claude cycle refrigerators with two or more stages of expansion. Since these refrigerators are helium liquefiers, cooling for gas cooled electrical leads can also be supplied by the helium refrigerator.

\section{THE DEVELOPMENT OF 4 K CRYOCOOLERS}

Small cryocoolers that can develop 4 to $5 \mathrm{~K}$ have been available for about thirty years. These coolers have all been three-stage coolers with the first two stages a Gifford McMahon (GM) cooler capable of generating 15 to $20 \mathrm{~K}$ at the cold head of the second stage. The third-stage is a separate Joule-Thompson (J-T) cooler that uses the first two stages to cool the helium for the final stage. The final temperature of $4.4 \mathrm{~K}$ comes from the J-T expansion. Historically the threestage coolers have not been very reliable, and in general they do not produce enough cooling to cool the superconducting magnet along with its electrical leads. These coolers do provide some cooling for shields.

The major change in cryocooler technology is the rare earth regenerators that make two-stage $4 \mathrm{~K} \mathrm{GM}$ and pulse tube coolers possible. This same regenerator material may eventually make a $4 \mathrm{~K}$ Sterling-cycle cooler practical. Closed circuit two-stage GM or pulse tube coolers are reliable with many hours of maintenance free of operation. A typical twostage GM or pulse tube cooler will develop 30 to $40 \mathrm{~W}$ of cooling at $50 \mathrm{~K}$, simultaneously with each watt of cooling at $4.2 \mathrm{~K}$. Both types of coolers are capable of generating temperatures in the $3 \mathrm{~K}$ range. The cooling at $50 \mathrm{~K}$ can be used for magnet thermal shields and current leads, provided there are low heat leak current leads between $50 \mathrm{~K}$ and $4 \mathrm{~K}$. No liquid nitrogen is needed for shield and lead cooling. 


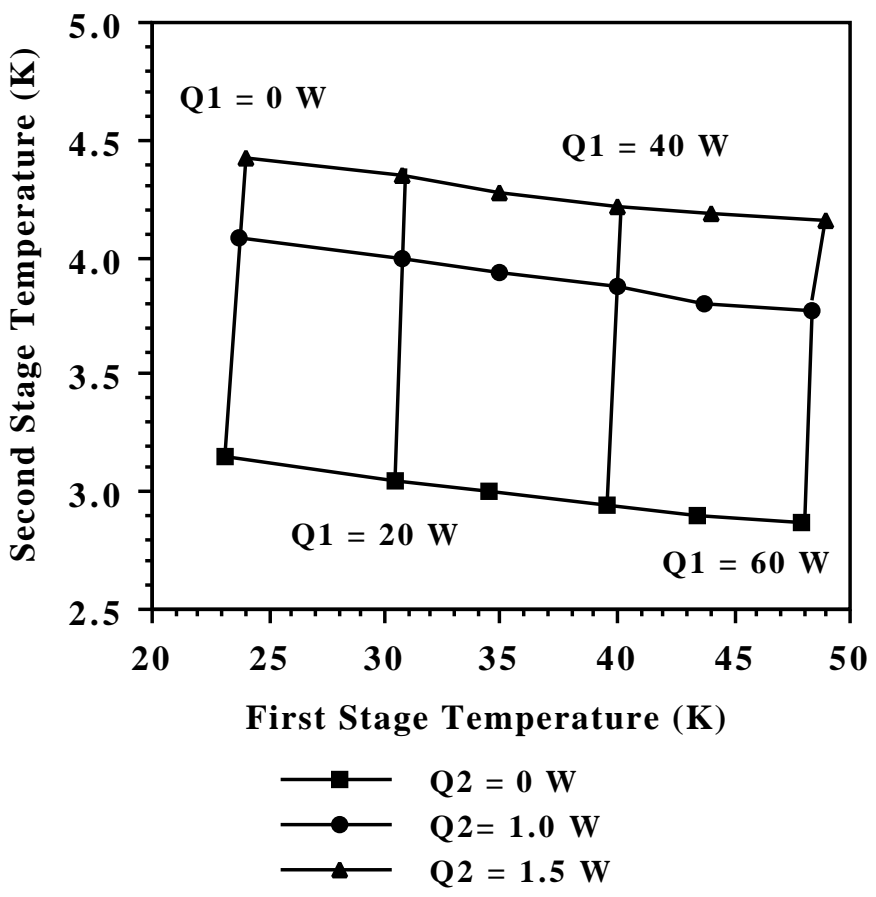

Figure 1. The Performance Curves for a $1.5 \mathrm{~W}$ (at $4.4 \mathrm{~K}$ ) Sumitomo SDRK 415 D Two-Stage GM Cryocooler
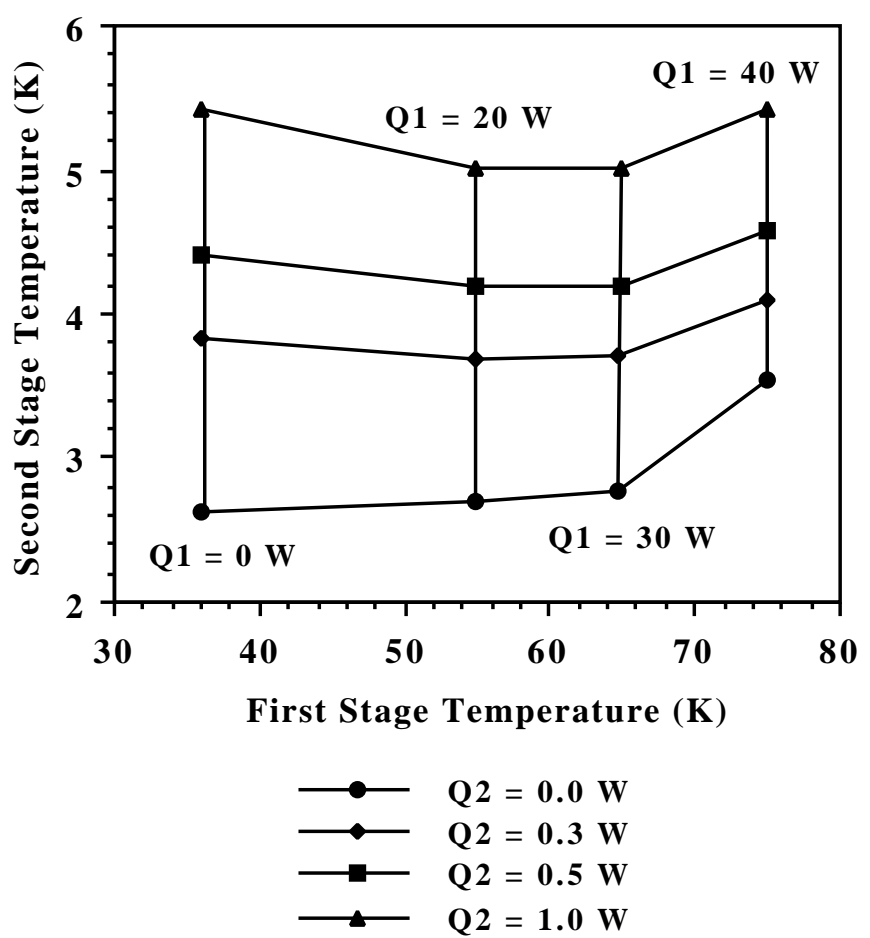

Figure 2. The Performance Curves for a $0.5 \mathrm{~W}$ (at $4.4 \mathrm{~K}$ ) Cryomech PT-405 Two-Stage Pulse Tube Cryocooler

Figure 1 shows the performance curves for the Sumitomo $1.5 \mathrm{~W}$ cryocooler[1]. A characteristic of this cooler is the second stage temperature goes down as more heat load is put on the first stage. Measurements by LBNL show that the
Sumitomo cryocooler behaves like the performance curves given in Figure 1[2]. Because the Sumitomo machine is a GM cooler, there is a moving piston system in the cold head. This moving piston does cause vibration that can be seen at the load. The cold heads should be vibration isolated from the load, when mechanical vibration is a detriment to magnet operation. Even though the cryocooler does vibrate (up to 1 gee based on cold head mass) and there are moving parts in the cold head, the machine is reliable with recommended service intervals of 10000 hours. A primary advantage of the $4 \mathrm{~K}$ pure GM cryocooler is the low temperatures achieved by the first stage of the cooler for a given heat load Q1 applied at the first stage.

The performance curves for the Cryomech $0.5 \mathrm{~W}$ pulse tube cooler is shown in Figure 2[3]. Like the GM cooler, the pulse tube cooler provides a lot of cooling at $50 \mathrm{~K}$, for each watt of cooling at $4.2 \mathrm{~K}$. The pulse tube cooler also likes to have a heat load on the first stage. When the stage two heat load Q2 is above about $0.15 \mathrm{~W}$, the second stage temperature goes down as heat is put into the first stage. The ratio of Q1/Q2 for the Cryomech pulse tube cooler is lower than for the Sumitomo GM cooler. This may not be characteristic of all pulse tube coolers, however. Pulse tube coolers have low vibration (up to 0.01 gee based on cold head mass), because there are no moving parts in the cold head. As a result, the recommended service interval for the Cryomech cooler is over 20000 hours. An apparent disadvantage of the pulse tube cooler is that it has a lower thermodynamic efficiency. The Cryomech $0.5 \mathrm{~W}$ pulse tube cooler requires a $5 \mathrm{~kW}$ compressor power to generate $0.5 \mathrm{~W}$ of cooling at $4.2 \mathrm{~K}$ plus $20 \mathrm{~W}$ at $55 \mathrm{~K}$. The Sumitomo $1.5 \mathrm{~W}$ GM cooler requires a $7.2 \mathrm{~kW}$ compressor power to generate $1.5 \mathrm{~W}$ of cooling at $4.2 \mathrm{~K}$ plus $60 \mathrm{~W}$ at $49 \mathrm{~K}$. At least part of this efficiency difference is due to the unit size.

It should be noted that other companies make cryocoolers. Leybolt makes a $0.25 \mathrm{~W}$ two-stage GM cooler. APD makes a $0.8 \mathrm{~W}$ GM cooler and $1.0 \mathrm{~W}$ cooler with a J-T circuit. Mitsubishi makes also make a GM cryocooler. Daiken Industries and CVI also make $4.4 \mathrm{~K}$ cryocoolers. There may be other suppliers of $4 \mathrm{~K}$ cryocoolers as well. It should be noted that the author does not endorse either of the two cryocoolers described in Figures 1 and 2 of this report. The readers of this paper should do their homework to determine which cryocooler is best for their magnet application.

\section{MAGNET TYPES COOLED WITH A 4 K CRYOCOOLER}

It is easy to define the types of magnets that should not be cooled using $4 \mathrm{~K}$ cryocoolers and they are: 1) large magnets with a cold mass in excess of 10 tons, 2) magnets with lead currents in excess of $2000 \mathrm{~A}, 3$ ) magnets that are part of a closely spaced string of magnets[4,5] (Many of the magnet applications in high energy accelerators fall into this category.), 4) any magnet that will have a radiation heat load larger than 1 watt during an extended period during its life time, and 5) magnets that have AC losses during charging and discharging that are more than a few watts. In general, most 
AC superconducting magnets such as SMES magnets, fusion magnets and pulsed magnets are not suitable to be cooled using a small $4 \mathrm{~K}$ cryocooler. Magnets that use liquid helium in the windings for stabilization are often not suitable for being cooled with small $4 \mathrm{~K}$ cryocoolers.

There are number of types of DC magnets that are well suited to be cooled using small cryocoolers. These magnets fall into the following broad categories: 1) single magnets used for NMR or MRI (provided their cold mass is less than about 5 tons), 2) small DC magnets used in any stand alone application with lead currents less than $600 \mathrm{~A}$, and 3) most stand alone magnets that operate in persistent mode (provided the magnet current is not above $1000 \mathrm{~A}$ ). Cooling magnets that operate in persistent mode using a small cryocooler can allow the leads to remain connected to the magnet at all times. This may be of advantage where rapid removal of the current from the magnet is needed for quench protection. Almost any small DC magnet that can be cooled using twophase helium in tubes can be cooled using a $4 \mathrm{~K}$ cryocooler, provided the magnet current is not too high.

The Lawrence Berkeley Laboratory is active pursuing the use of cryocoolers for superconducting magnets for the following applications: 1) the three superconducting dipoles in the ALS ring will be cooled using $1.5 \mathrm{~W}$ cryocoolers[6]; 2) a mixed superconducting sextupole and solenoid for an ion source for the 88 inch cyclotron will be cooled using a pair of $1.5 \mathrm{~W}$ coolers[7]; 3) cryocoolers will probably be used to cool test magnets for the neutrino factory; 4) a test multi-bore quadrupole for heavy ion fusion can be cooled using cryocoolers; 5) a proposed superconducting undulator magnet for the ALS light source can be cooled using a $4.2 \mathrm{~K}$ cryocooler (probably a low vibration pulse tube cooler); and 6) a proposed superconducting gradient magnet for measuring the thickness and moisture content of paper being processed in a paper mill can be cooled using a $4.2 \mathrm{~K}$ cryocooler. The paper mill application is an application where conventional superconducting magnet cooling systems are completely unacceptable to the industry. Other superconducting magnet applications for cryocoolers remain to be explored.

\section{CRYOCOOLER COOLED MAGNET CONFIGURATIONS}

The key to cooling niobium titanium magnets with a cryocooler is the development of HTS leads that operate between the first stage temperature and the second stage temperature of the cryocooler. These leads allow a magnet to be continuously powered and they will greatly reduce the heat load into the $4 \mathrm{~K}$ region without having to retract the leads. In general, the heat flow $\mathrm{Q}_{\mathrm{L} 2}$ down HTS leads can be estimated using the following expression:

$$
\mathrm{Q}_{\mathrm{L} 2}=0.00025 \mathrm{I}\left[\mathrm{T}_{1} / 40\right]^{2}
$$

where $\mathrm{I}$ is the magnet maximum design current; and $\mathrm{T}_{1}$ is the temperature of the first stage of the cryocooler. Cryocoolers can not provide cold helium gas for gas cooled electrical leads, so the lead heat leak must be conducted down the upper leads to the first stage of the cryocooler. For a continuously powered magnet the lead heat flow to the first stage of the cryocooler $\mathrm{Q}_{\mathrm{L} 1}$ is given by the following expression:

$$
\mathrm{Q}_{\mathrm{L} 1}=0.065 \mathrm{I}
$$

Equations 1 and 2 explain why the cryocooler must often be sized for the heat load going into the cryocooler first stage. Magnets that charge quickly and operate in persistent mode can have bronze leads that carry current between room temperature and the top of the HTS leads, which will reduce the heat flow to the cooler first stage when the magnet is persistent. During charging, the cryocooler first stage temperature will rise, but when the magnet is put into persistent mode, both the first and second stage cryocooler temperatures will fall to their normal operating level. The HTS leads must be designed to operate at a temperature that is at least $10 \mathrm{~K}$ above their highest operating temperature.

Figure 3 is schematic representation of a superconducting magnet its shields and leads that are cooled using a two stage cryocooler. The first stage intercepts heat flowing from room temperature through the cryostat insulation, through the cold mass supports, through instrumentation wires and through solid metal leads that carry current into the magnet. The first stage heat load is dominated by the leads. The second stage removes heat flowing into the $4 \mathrm{~K}$ region by radiation, by conduction down the cold mass supports and instrumentation wires and by conduction down HTS leads between the first stage of the cryocooler and the magnet. In most cases, the HTS leads are the dominant heat loads into the $4 \mathrm{~K}$ region. The next largest $4 \mathrm{~K}$ heat load is the cold mass supports.

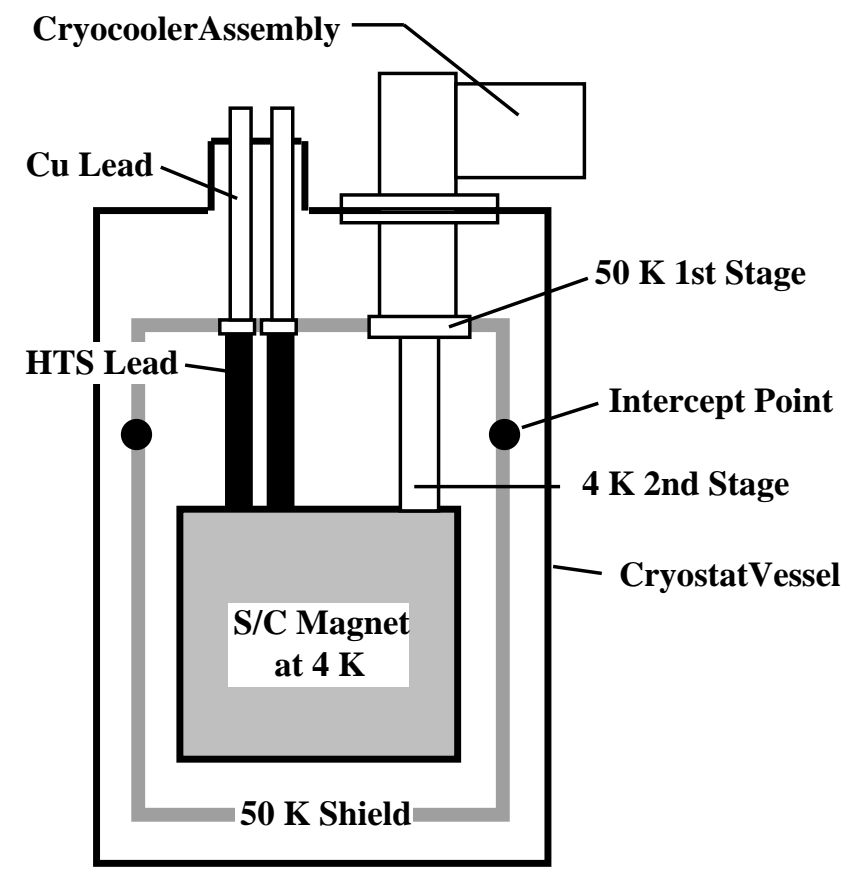

Figure 3. A Schematic Diagram of a Superconducting Magnet, Its Shields and Leads Cooled with a Two Stage $4 \mathrm{~K}$ Cryocooler 
Figure 3 shows no liquid cryogen reservoirs or any liquid cryogen cooling of any kind. A magnet cooled using a cryocooler can be cooled entirely by conduction provided there is a very low thermal resistance path between the coil and the cryocooler. Cryocoolers can be used to cool down the superconducting magnets down from $300 \mathrm{~K}$ to $4 \mathrm{~K}$. One must allow time for the cryocooler to cool the magnet down, if the cryocooler is used alone. As a rule of thumb, a cryocooler that generates $1 \mathrm{~W}$ at $4.2 \mathrm{~K}$ will need about one month to cool $1000 \mathrm{~kg}$ of mass from $300 \mathrm{~K}$ to $4 \mathrm{~K}$. Large magnets or magnets with cold iron will often have pipes that can used to carry liquid nitrogen and liquid helium to cool the cold mass down quickly. Once the magnet has been cooled to near $10 \mathrm{~K}$, the cool down lines must be evacuated. The cryocooler can finish the magnet cool down.

In general, a cryocooler is used to cool superconducting magnets that are fabricated from potted coils. Potted coils are cooled by conduction even if they are in a bath of liquid helium. Liquid helium is not necessary anywhere within the cryostat when a cryocooler is used to cool the magnet. A $4 \mathrm{~K}$ cryocooler is not a helium liquefier. The only way that helium can be liquefied is by condensation on the stage 2 cold head, provided it is in an insulated tank that can contain the liquid helium. Liquid nitrogen is also unnecessary when a cryocooler is used to cool a magnet. In general, the first stage cold head operates at a temperature that is substantially lower than $77 \mathrm{~K}$. Experiments on the ALS Superbend test magnet show that the presence of liquid helium has no effect on magnet performance[6]. The maximum safe charging rate for the magnet is the same with or without liquid helium in the tank attached to the magnet iron and the cryocooler.

There are two arguments for liquid helium and liquid nitrogen in a magnet system that is cooled using a cryocooler. The first is that liquid helium will permit the magnet to remain cold during an extended power shut down and even during a failure of the cryocooler itself. Accelerators may require this level of redundancy. If one wants to keep the magnet cold for a number of hours with the cryocooler shut off, one must have both liquid helium and nitrogen vessels attached to the cryocooler and the magnet. A second argument for liquid helium in a magnet cooled with a $4 \mathrm{~K}$ cryocooler is having a buffer for the heat generated by rapid charging and discharging of the superconducting magnet.

\section{TABLE 1. THE S UPERBEND DIPOLE DESIGN HEAT LOADS WITH AND WITHOUT THE 1.5 W CRYOCOOLER}

\section{Parameter}

Parasitic Heat Leaks with Cooler On (W)

Heat Leaks down the Leads (W)

Stage Heat Load with Cooler On (W)

Stage Temperature with Cooler On (K)

Heat Flow down Cooler Stage Off (W)

Stage Heat Load with Cooler Off (W)

Stage Temperature with Cooler Off $(\mathrm{K})$

Cryogen Boil-off w/o Cooler (1/hr)

$\begin{array}{cc}\text { Stage 1 } & \text { Stage 2 } \\ 11.5 & 0.193 \\ 27.0 & 0.250 \\ 38.5 & 0.423 \\ 39.0 & 3.4 \\ \sim 50 & \sim 2 \\ \sim 90 & \sim 2.4 \\ 77.5 & 4.3 \\ \sim 2.0 & \sim 4.0\end{array}$

Table 1 shows the design heat loads for the Superbend dipole. The dipole has a cold mass of $1700 \mathrm{~kg}$ and a current of 300 A. Table 1 illustrates that the Superbend dipole can be made to operate on a cryocooler with an emergency liquid cryogen backup, for redundancy. For most applications where the use of a $4 \mathrm{~K}$ cryocooler is attractive, no liquid helium or liquid nitrogen backup is needed.

\section{CONCLUDING COMMENTS}

The use of $4 \mathrm{~K}$ cryocoolers permits one to build reliable superconducting magnets that may be cost competitive with conventional magnets. The cost of the niobium titanium coils plus a $4 \mathrm{~K}$ cryocooler can be substantially lower than coils made with HTS conductor cooled plus a 20 to $30 \mathrm{~K}$ cryocooler.

The use of $4 \mathrm{~K}$ cryocooler permits one to operate a variety of magnets in a stand alone cryogen free environment. Magnets cooled with $4 \mathrm{~K}$ cryocooler must be housed in low heat leak cryostat. The reduced first temperature of the cryocooler makes a low heat leak cryostat relatively easy to build. HTS electrical leads between the first and second stages of the cryocooler are necessary. The HTS leads permit the superconducting magnet to be operated with continuos current flow through the leads. Persistent magnets that are cooled using a $4 \mathrm{~K}$ cryocooler can be operated with their leads connected.

There are limits to the use of $4 \mathrm{~K}$ cryocoolers; they do not work well when the magnet cold mass is over 5 tons or when the lead current is over 600 to $1000 \mathrm{~A}$. In general, the heat going down the leads into the magnet limits the temperature of both stages of the cryocooler.

\section{REFERENCES}

[1] The Sumitomo Data Sheet for the SRDK-415D cryocooler from Janis Research Inc., Wilmington MA 01887 (Janis is the US seller of Sumitomo cryocoolers.)

[2] J. Zbasnik et al, "Tests of a GM Cryocooler and High Tc Leads for Use on the ALS Superbend Magnets," to be published Advances in Cryogenic Engineering 45, Plenum Press, NY (1999)

[3] P. Gifford and C. Wnag, Cryomech Inc. Syracuse, NY 13211, private communication concerning the PT-405 pulse tube cryocooler and its performance, (August 2000)

[4] M. A. Green et al, "Refrigeration Options for the Advanced Light Source Superbend Dipole Magnets," to be published in Advances in Cryogenic Engineering 45, Plenum Press NY (1999)

[5] M. A. Green, J. Fockler, R. E. Lafever, D. L. Vanecek and S. S. Yu, "A Thin Superconducting Solenoid for Use in a Phase Rotation Induction Linac," this proceedings, IEEE Trans. on Applied Superconductivity 11, (2001)

[6] J. DeVries et al, "ALS Superbend Magnet System Design," this proceedings, IEEE Trans. on Applied Superconductivity 11, (2001)

[7] C. Taylor et al, "Magnet System for an ECR Ion Source," IEEE Trans. on Applied Superconductivity 1 0, No. 1, p 224, (2000) 